\title{
SOBOLEV SPACES ON METRIZABLE GROUPS
}

\section{Przemysław Górka and Tomasz Kostrzewa}

\author{
Warsaw University of Technology, Department of Mathematics and Information Sciences \\ Ul. Koszykowa 75, 00-662 Warsaw, Poland; pgorka@mini.pw.edu.pl \\ Warsaw University of Technology, Department of Mathematics and Information Sciences \\ Ul. Koszykowa 75, 00-662 Warsaw, Poland; kostrzewat@mini.pw.edu.pl
}

\begin{abstract}
We continue our research on Sobolev spaces on locally compact abelian groups. In this paper, we focus on embedding results when the dual group is metrizable. We show the density of continuous functions. As a by-product we obtain new embeddings of Sobolev spaces defined over $p$-adic numbers.
\end{abstract}

\section{Introduction}

The purpose of this paper is to continue the investigation of Sobolev spaces on locally compact abelian (LCA) groups. Sobolev spaces are well understood on (domains of) $\mathbf{R}^{n}$, see [1, 27], compact Riemannian manifolds [4, 15], and metric measure spaces $[14,26]$. There are also some works on Sobolev spaces in the $p$-adic context, see $[18,24]$ and references therein, and in special cases of locally compact groups such as the Heisenberg group (see [5, 7]).

We introduced Sobolev spaces on LCA groups in [13]. In that reference we proved analogues of the Sobolev embedding and Rellich-Kondrachov theorems, and we used these results to prove the existence of regular solutions to some equation of infinite order. In [12] we improved previous theorems about continuous and compact embeddings (see also [11] for related result in the case of countable dual groups). Moreover in [12], we introduced Sobolev spaces on subsets of LCA groups and we proved analogue of Rellich lemma for those spaces. In our paper we study Sobolev spaces on locally compact abelian groups in the case when the dual group is metrizable.

The remainder of the paper is organized as follows. In Preliminaries we review some definitions from harmonic analysis and present the theory of Sobolev spaces on topological groups. Moreover, some general remarks about metrizable groups are given. Then, in Section 3 we prove various embeddings type theorems for Sobolev spaces on locally compact abelian groups. Namely, we establish the embedding into $L^{p}$ as well as into Hölder spaces. Furthermore, the so-called Moser-Trudinger inequality is proved. In that section we assume that the dual group $G^{\wedge}$ is metrizable and the measure on $G^{\wedge}$ is upper $\beta$-regular (see Preliminaries for the definition). Section 4 is devoted to studying the density of continuous functions in Sobolev space. In Theorem 5 we show that the space of continuous functions is dense in the Sobolev space defined on any LCA groups. Finally, let us stress that most of the results presented in this paper can be applied to the Sobolev spaces over $p$-adic numbers.

doi:10.5186/aasfm.2015.4045

2010 Mathematics Subject Classification: Primary 32A99, 46E35, 22B99.

Key words: Abstract harmonic analysis, Sobolev spaces, analysis on metric measure spaces, $p$-adic analysis, embeddings, Moser-Trudinger inequality. 


\section{Preliminaries}

We use standard notations from harmonic analysis (see [8] and [17]). Let us fix a locally compact abelian group $G$. We denote by $\mu_{G}$ the unique Haar measure of $G$ and by $G^{\wedge}$ the dual group of the group $G$ that is, $G^{\wedge}$ is the locally compact abelian group of all continuous group homomorphisms from $G$ to the circle group $\mathbf{T}$. We denote by $\hat{\mu}_{G}$ Haar measure (Plancherel measure) on $G^{\wedge}$. By $e$ (resp. $\hat{e}$ ) we shall denote the identity of $G$ (resp. $\hat{G}$ ). The $L^{p}$ spaces over $G$ are defined as usual,

$$
L^{p}(G)=\left\{f: G \rightarrow \mathbf{C}: \int_{G}|f(x)|^{p} d \mu_{G}(x)<\infty\right\},
$$

and we set

$$
\|f\|_{L^{p}(G)}=\left(\int_{G}|f(x)|^{p} d \mu_{G}(x)\right)^{1 / p} .
$$

Since the definition of the Sobolev space on locally compact abelian group uses essentially the Fourier transform, we also recall that the Fourier transform on $G$ is defined as follows: if $f \in L^{1}(G)$, then it Fourier transform is the function $\hat{f}: G^{\wedge} \rightarrow \mathbf{C}$ given by

$$
\hat{f}(\xi)=\int_{G} \overline{\xi(x)} f(x) d \mu_{G}(x) .
$$

Now, let us recall the notion of the Sobolev spaces on locally compact abelian groups (see [13], [11] and [12]).

Definition 1. Let us fix a map $\gamma: G^{\wedge} \rightarrow[0, \infty)$ and a nonnegative real number $s$. We shall say that $f \in L^{2}(G)$ belongs to the Sobolev space $H_{\gamma}^{s}(G)$ if the following integral is finite:

$$
\int_{G^{\wedge}}\left(1+\gamma(\xi)^{2}\right)^{s}|\hat{f}(\xi)|^{2} d \hat{\mu}_{G}(\xi)
$$

Moreover, for $f \in H_{\gamma}^{s}(G)$ its norm $\|f\|_{H_{\gamma}^{s}(G)}$ is defined as follows:

$$
\|f\|_{H_{\gamma}^{s}(G)}=\left(\int_{G^{\wedge}}\left(1+\gamma(\xi)^{2}\right)^{s}|\hat{f}(\xi)|^{2} d \hat{\mu}_{G}(\xi)\right)^{\frac{1}{2}} .
$$

For the basic properties of this kind of spaces we refer to [13].

We now turn to a brief presentation of the theory of metrizable locally compact groups. Let us recall that the Birkhoff-Kakutani theorem [20] states that the LCA group is metrizable if and only if it is first countable. On the other hand, if LCA group $G$ is the second countable, then there exists an invariant metric (i.e. $d(x, y)=d(g x, g y)$ for all $x, y, g \in G)$ in which all balls are relatively compact (see [25]). Furthermore, if $G$ is LCA and second countable, then the dual group $G^{\wedge}$ is second countable too (see $[6]$ ).

Definition 2. Let $\mathrm{G}$ be a LCA group. We say that metrics $d: G \times G \rightarrow \mathbf{R}_{+}$and $\hat{d}: G^{\wedge} \times G^{\wedge} \rightarrow \mathbf{R}_{+}$are dual metrics if for each character $\xi \in G^{\wedge}$ and every $x, y \in G$ we have

$$
|\xi(x)-\xi(y)| \leq \hat{d}(\xi, \hat{e}) d(x, y)
$$


Example 1. The groups $\mathbf{R}^{n}, \mathbf{T}^{n}$ and $\mathbf{Q}_{p}^{n}$ satisfy condition of the above definition $^{1}$.

The condition in Definition 2 is a Lipschitz continuity of characters. Note that in general, for locally compact groups with invariant metric we can only conclude that characters are uniformly continuous.

Propositon 1. Let $G$ be a locally compact group with invariant metric, then every character is uniformly continuous.

Probably, this observation is well known, but for the sake of completeness we include the proof.

Proof. Let us fix the character $\xi \in G^{\wedge}$. From the continuity of $\xi$, for any $\varepsilon>0$, there exists $\delta>0$ such that

$$
|\xi(e)-\xi(z)|<\varepsilon
$$

when $d(e, z)<\delta$. Next, taking $x, y \in G$ such that $d(x, y)=d\left(e, x^{-1} y\right)<\delta$, we get

$$
|\xi(x)-\xi(y)|=|\xi(x)|\left|\xi(e)-\xi\left(x^{-1} y\right)\right|=\left|\xi(e)-\xi\left(x^{-1} y\right)\right|<\varepsilon .
$$

Let us mention a very interesting consequence of the above definition.

Propositon 2. Let $G$ be a $L C A$ group such that $d$ and $\hat{d}$ are dual metrics. If $G$ is second countable and $G^{\wedge}$ is bounded, then $G$ is countable.

Proof. First note that $G^{\wedge} \subset C(G, \mathbf{T})$. Since $\mathbf{T}$ is compact, it follows that for all $a \in G$ the set

$$
G_{a}^{\wedge}=\left\{\xi(a): \xi \in G^{\wedge}\right\} \subset \mathbf{T}
$$

has a compact closure. Moreover, by the assumption on $\hat{d}$ we have that $G^{\wedge}$ is equicontinuous. Hence, from the Arzela-Ascoli theorem (see e.g. [22]) we conclude that $G^{\wedge}$ is relatively compact in $C(G, \mathbf{T})$. On the other hand, since $G^{\wedge}$ is closed, we get that $G^{\wedge}$ is compact. Thus, by the Pontryagin duality, we have that $G$ is discrete. Finally, it follows that $G$ must be countable.

The following definition will be needed throughout the paper.

Definition 3. Let $(G, d, \mu)$ be a LCA group with metric $d$ and the Haar measure $\mu$. We shall say that the measure $\mu$ is upper $\beta$-regular if:

1. $G$ is not discrete and there exists a constant $D_{\beta}>0$, such that for all $r>0$ we have

$$
\mu(B(e, r)) \leq D_{\beta} r^{\beta}
$$

2. $G$ is discrete and there exists $R_{0}>0$ and $D_{\beta}>0$ such that $B\left(e, R_{0}\right)=\{e\}$ and for $r \geq R_{0}$

$$
\mu(B(e, r)) \leq D_{\beta} r^{\beta} .
$$

Remark 1. The degree $\beta$ in Definition 3 can be treated as an analogue of the dimension for groups. Similar condition appears in the analysis on metric measure spaces (see $[16,14])$.

Remark 2. Assume that $\left(G^{\wedge}, \hat{d}\right)$ is discrete and $\hat{\mu}_{G}$ is upper $\beta$-regular. Then, from the Pontryagin duality $(G, d)$ is compact and thus bounded. Since in the above

\footnotetext{
${ }^{1}$ Basic properties of the $p$-adic world can be found in the monograph [23].
} 
definition $R_{0}$ is not unique, we shall always assume that

$$
R_{0} \leq \min \left(\frac{1}{\sup _{x, y \in G} d(x, y)}, 1\right) .
$$

The following property allows us to extend our considerations to the cases when $\gamma(\xi) \neq \hat{d}(\xi, \hat{e})$. Nevertheless, the most important case is when $\gamma(\xi)=\hat{d}(\xi, \hat{e})$.

Definition 4. Let $G$ be a LCA group and $\omega, A>0$. We say that a function $\gamma: G^{\wedge} \rightarrow[0,+\infty)$ belongs to $\Gamma_{A}(\omega)$ if for all $\xi \in G^{\wedge}$ the following inequality holds

$$
A \hat{d}(\xi, \hat{e})^{\omega} \leq \gamma(\xi)
$$

From now on, we write $d$ for metric on $G$ as well as $\hat{d}$ for metric on $G^{\wedge}$. Moreover, for simplicity of notation, we write $C_{X, \delta}$ instead of $\max \left(1, X^{-2 \delta}\right)$.

\section{Embedding theorems}

We discuss in this chapter the validity of Sobolev embeddings. We shall assume that the dual group $G^{\wedge}$ is metrizable and the measure $\hat{\mu}$ is upper $\beta$-regular. Let us mention that in the theory of Sobolev spaces on metric measure spaces it is frequently assumed that the measure $\mu$ on metric space $(X, d)$ is lower $\beta$-regular (see $[3,14,16]$ ) . On the other hand, we deal with dual objects such as $G^{\wedge}$ and $\hat{\mu}$, thus our assumption on the measure $\hat{\mu}$ is dual in a sense.

3.1. Embedding into $\boldsymbol{L}^{p}$-spaces. We start with the following extremely useful lemma.

Lemma 1. Let $G$ be a LCA group. Suppose that $\gamma \in \Gamma_{A}(\omega)$ and the measure $\hat{\mu}$ is upper $\beta$-regular. If $\alpha>\frac{\beta}{2 \omega}$, then $\left(1+\gamma^{2}(.)\right)^{-1} \in L^{\alpha}\left(G^{\wedge}\right)$. Moreover, for every $R>0$ the following inequality

$$
\left\|\frac{1}{1+\gamma^{2}(.)}\right\|_{L^{\alpha}\left(G^{\wedge}\right)}^{\alpha} \leq C_{A, \alpha} D_{\beta}\left(\max \left(\frac{\hat{\mu}_{G}(\{\hat{e}\})}{D_{\beta}}, R^{\beta}\right)+\frac{2^{\beta} R^{\beta-2 \omega \alpha}}{1-2^{\beta-2 \omega \alpha}}\right)
$$

holds.

Proof. Let us fix $\alpha>\frac{\beta}{2 \omega}$ and $R>0$. It is easy to see that

$$
\frac{1}{\left(1+\gamma^{2}(\xi)\right)^{\alpha}} \leq \frac{C_{A, \alpha}}{\left(1+\hat{d}^{2 \omega}(\xi, \hat{e})\right)^{\alpha}}
$$

Next, we shall consider two cases:

1. $G^{\wedge}$ is not discrete. Since $\beta-2 \omega \alpha<0$, we have

$$
\begin{aligned}
& \int_{G^{\wedge}} \frac{d \hat{\mu}_{G}(\xi)}{\left(1+\hat{d}(\xi, \hat{e})^{2 \omega}\right)^{\alpha}}=\int_{B(\hat{e}, R)} \frac{d \hat{\mu}_{G}(\xi)}{\left(1+\hat{d}(\xi, \hat{e})^{2 \omega}\right)^{\alpha}}+\int_{G^{\wedge} \backslash B(\hat{e}, R)} \frac{d \hat{\mu}_{G}(\xi)}{\left(1+\hat{d}(\xi, \hat{e})^{2 \omega}\right)^{\alpha}} \\
& \leq D_{\beta} R^{\beta}+\sum_{k=0}^{\infty} \int_{B\left(\hat{e}, 2^{k+1} R\right) \backslash B\left(\hat{e}, 2^{k} R\right)} \frac{d \hat{\mu}_{G}(\xi)}{\left(1+\hat{d}(\xi, \hat{e})^{2 \omega}\right)^{\alpha}} \leq D_{\beta} R^{\beta}+D_{\beta} \sum_{k=0}^{\infty} \frac{\left(2^{k+1} R\right)^{\beta}}{2^{2 \omega \alpha k} R^{2 \omega \alpha}} \\
& =D_{\beta}\left(R^{\beta}+2^{\beta} R^{\beta-2 \omega \alpha} \sum_{k=0}^{\infty}\left(2^{\beta-2 \omega \alpha}\right)^{k}\right)=D_{\beta}\left(R^{\beta}+\frac{2^{\beta} R^{\beta-2 \omega \alpha}}{1-2^{\beta-2 \omega \alpha}}\right) .
\end{aligned}
$$


2. $G^{\wedge}$ is discrete. If $R \geq R_{0}$, then the proof runs as before. Now, for $R<R_{0}$ we have

$$
\begin{aligned}
\int_{G^{\wedge}} \frac{d \hat{\mu}_{G}(\xi)}{\left(1+\hat{d}(\xi, \hat{e})^{2 \omega}\right)^{\alpha}} & \leq \hat{\mu}_{G}(\{\hat{e}\})+\int_{G^{\wedge} \backslash B(\hat{e}, R)} \frac{d \hat{\mu}_{G}(\xi)}{\left(1+\hat{d}(\xi, \hat{e})^{2 \omega}\right)^{\alpha}} \\
& =\hat{\mu}_{G}(\{\hat{e}\})+\int_{G^{\wedge} \backslash B\left(\hat{e}, R_{0}\right)} \frac{d \hat{\mu}_{G}(\xi)}{\left(1+\hat{d}(\xi, \hat{e})^{2 \omega}\right)^{\alpha}} \\
& \leq \hat{\mu}_{G}(\{\hat{e}\})+D_{\beta} \frac{2^{\beta} R_{0}^{\beta-2 \omega \alpha}}{1-2^{\beta-2 \omega \alpha}} \\
& \leq \hat{\mu}_{G}(\{\hat{e}\})+D_{\beta} \frac{2^{\beta} R^{\beta-2 \omega \alpha}}{1-2^{\beta-2 \omega \alpha}} .
\end{aligned}
$$

This completes the proof.

Now, we are in position to state the main result of this subsection.

Theorem 1. Let $G$ be a LCA group. Suppose that $\gamma \in \Gamma_{A}(\omega)$, the measure $\hat{\mu}$ is upper $\beta$-regular and $0<s<\frac{\beta}{2 \omega}$. Then for all $\alpha>\frac{\beta}{2 \omega}$ the following embedding holds

$$
H_{\gamma}^{s}(G) \hookrightarrow L^{\alpha^{*}}(G),
$$

where $\alpha^{*}=\frac{2 \alpha}{\alpha-s}$. Moreover, there exists a constant $C>0$ such that for each $u \in$ $H_{\gamma}^{s}(G)$ we have

$$
\|u\|_{L^{\alpha^{*}}(G)} \leq C\|u\|_{H_{\gamma}^{s}(G)} .
$$

Furthermore, if additionally $G$ is compact and $p<\alpha^{*}$, then

$$
H_{\gamma}^{s}(G) \hookrightarrow \hookrightarrow L^{p}(G),
$$

where $\hookrightarrow \hookrightarrow$ denotes the compact embedding.

Proof. By virtue of Theorem 3.5 from [13], it is sufficient to show that $\frac{1}{1+\gamma^{2}(\cdot)} \in$ $L^{\alpha}\left(G^{\wedge}\right)$. Let us fix $R>0$. Hence, from Lemma 1 we have

$$
\left\|\frac{1}{1+\gamma^{2}(.)}\right\|_{L^{\alpha}}^{\alpha} \leq C_{A, \alpha} D_{\beta}\left(\max \left(\frac{\hat{\mu}_{G}(\{\hat{e}\})}{D_{\beta}}, R^{\beta}\right)+\frac{2^{\beta} R^{\beta-2 \omega \alpha}}{1-2^{\beta-2 \omega \alpha}}\right)<\infty .
$$

This is our claim. On the other hand, the compactness follows from Theorem 11 in [12].

We finish this subsection with a very important consequence of the above theorem.

Corollary 1. Let $G$ be a LCA group. Suppose that $\gamma(\xi)=\hat{d}(\xi, \hat{e})$, the measure $\hat{\mu}$ is upper $\beta$-regular. If $0<s<\frac{\beta}{2}$, then for all $\alpha>\frac{\beta}{2}$ the following embedding holds

$$
H_{\gamma}^{s}(G) \hookrightarrow L^{\alpha^{*}}(G),
$$

where $\alpha^{*}=\frac{2 \alpha}{\alpha-s}$. Moreover, there exists a constant $C>0$ such that for each $u \in$ $H_{\gamma}^{s}(G)$ we have

$$
\|u\|_{L^{\alpha^{*}}(G)} \leq C\|u\|_{H_{\gamma}^{s}(G)} .
$$

3.2. Embedding into Hölder spaces. In this section we shall assume that the group and its dual group are both metrizable. Before the formulation our next result 
we recall the notion of Hölder spaces. Namely, we shall say that a continuous and bounded function $u$ belongs to the Hölder space $C^{0, \alpha}(G)$ if the following seminorm

$$
\sup _{x \neq y \in G} \frac{|u(x)-u(y)|}{d(x, y)^{\alpha}}
$$

is finite. $C^{0, \alpha}(G)$ is a Banach space with respect to the following norm

$$
\|u\|_{C^{0, \alpha}(G)}=\|u\|_{C(G)}+\sup _{x \neq y \in G} \frac{|u(x)-u(y)|}{d(x, y)^{\alpha}} .
$$

Theorem 2. Let $G$ be a LCA group such that $d$ and $\hat{d}$ are dual metrics and $\hat{\mu}$ is upper $\beta$-regular. Additionally, let us assume that $\gamma \in \Gamma_{A}(\omega)$ and that $\omega s=$ $\alpha+\frac{\beta}{2}$ for some $\alpha \in(0,1)$. Then, $H_{\gamma}^{s}(G) \hookrightarrow C^{0, \alpha}(G)$. Moreover, there exists $C=$ $C\left(s, \omega, \beta, A, D_{\beta}\right)$ such that the inequality

$$
\|u\|_{C^{0, \alpha}(G)} \leq C\|u\|_{H_{\gamma}^{s}(G)}
$$

holds for all $u \in H_{\gamma}^{s}(G)$. Furthermore, if $G$ is compact and $\lambda<\alpha$, then

$$
H_{\gamma}^{s}(G) \hookrightarrow \hookrightarrow C^{0, \lambda}(G)
$$

Proof. First of all let us note that $s>\frac{\beta}{2 \omega}$, hence from Lemma $1\left(1+\gamma^{2}(.)\right)^{-1} \in$ $L^{s}\left(G^{\wedge}\right)$. Thus, it follows from Theorem 3.3 in [13] that $H_{\gamma}^{s}(G) \hookrightarrow C(G)$.

Now, we shall show that $u$ is Hölder continuous. For almost all $x, y \in G$ such that $x \neq y$, by the Cauchy-Schwartz inequality we get

$$
|u(x)-u(y)| \leq \sqrt{\int_{G^{\wedge}} \frac{|\xi(x)-\xi(y)|^{2}}{\left(1+\gamma^{2}(\xi)\right)^{s}} d \hat{\mu}_{G}(\xi)} \cdot\|u\|_{H_{\gamma}^{s}(G)} .
$$

Next, let us set $r=d(x, y)$, then we get

$$
\begin{aligned}
& \int_{G^{\wedge}} \frac{|\xi(x)-\xi(y)|^{2}}{\left(1+\gamma^{2}(\xi)\right)^{s}} d \hat{\mu}_{G}(\xi) \\
& \leq \int_{B\left(\hat{e}, \frac{1}{r}\right)} \frac{|\xi(x)-\xi(y)|^{2}}{\left(1+\gamma^{2}(\xi)\right)^{s}} d \hat{\mu}_{G}(\xi)+4 C_{A, s} \int_{G^{\wedge} \backslash B\left(\hat{e}, \frac{1}{r}\right)} \frac{d \hat{\mu}_{G}(\xi)}{\left(1+\hat{d}(\xi, \hat{e})^{2 \omega}\right)^{s}}=I_{1}+4 C_{A, s} I_{2} .
\end{aligned}
$$

Using the methods explored in the proof of Lemma 1 we get

$$
I_{2} \leq D_{\beta} \cdot 2^{\beta}\left(\frac{1}{r}\right)^{-2 \alpha} \sum_{n=0}^{\infty}\left(2^{-2 \alpha}\right)^{n}=D_{\beta} \frac{2^{2 \alpha+\beta} r^{2 \alpha}}{2^{2 \alpha}-1} .
$$

Subsequently, we shall show that

$$
I_{1} \leq C_{A, s} D_{\beta} \frac{2^{2 \omega s}}{1-2^{2 \alpha-2}} r^{2 \alpha} .
$$

When $G^{\wedge}$ is not a discrete group, then we have $\hat{\mu}_{G}(\{\hat{e}\})=0$. Thus

$$
\int_{\{\hat{e}\}} \frac{|\xi(x)-\xi(y)|^{2}}{\left(1+\gamma^{2}(\xi)\right)^{s}} d \hat{\mu}_{G}(\xi)=0 .
$$

On the other hand, if $G^{\wedge}$ is a discrete group, then one can easily convince oneself that

$$
\int_{B\left(\hat{e}, R_{0}\right)} \frac{|\xi(x)-\xi(y)|^{2}}{\left(1+\gamma^{2}(\xi)\right)^{s}} d \hat{\mu}_{G}(\xi)=0 .
$$


According to the above observation and since $d$ and $\hat{d}$ are dual metrics, the term $I_{1}$ can be estimated in the following manner

$$
\begin{aligned}
I_{1} & \leq C_{A, s} \sum_{k=0}^{\infty} \int_{B\left(\hat{e}, \frac{1}{2^{k_{r}}}\right) \backslash B\left(\hat{e}, \frac{1}{2^{k+1}}\right)} \frac{\hat{d}(\xi, \hat{e})^{2} r^{2}}{\left(1+\hat{d}(\xi, \hat{e})^{2 \omega}\right)^{s}} d \hat{\mu}_{G}(\xi) \\
& \leq C_{A, s} D_{\beta} \sum_{k=0}^{\infty} \frac{2^{-2 k} r^{-2} r^{2}}{2^{-(k+1) 2 \omega s} r^{-2 \omega s}} 2^{-k \beta} r^{-\beta} \\
& =2^{2 \omega s} r^{2 \alpha} C_{A, s} D_{\beta} \sum_{k=0}^{\infty}\left(2^{2 \alpha-2}\right)^{k}=C_{A, s} D_{\beta} \frac{2^{2 \omega s}}{1-2^{2 \alpha-2}} r^{2 \alpha} .
\end{aligned}
$$

Recalling that $r=d(x, y)$ and gathering estimates (3), (2) and (1) we get

$$
\frac{|u(x)-u(y)|}{d(x, y)^{\alpha}} \leq \sqrt{C_{A, s} D_{\beta}} \sqrt{\frac{2^{2 \omega s}}{1-2^{2 \alpha-2}}+\frac{2^{2 \omega s+2}}{2^{2 \alpha}-1}}\|u\|_{H_{\gamma}^{s}(G)} .
$$

Finally, using the embedding $H_{\gamma}^{s}(G) \hookrightarrow C(G)$ and the above inequality, we conclude that there exists a constant $C=C\left(s, \omega, \beta, A, D_{\beta}\right)$ such that

$$
\|u\|_{C^{0, \alpha}(G)} \leq C\|u\|_{H_{\gamma}^{s}(G)},
$$

and the proof of the continuity of the embedding is complete. Now, the routine application of the Arzela-Ascoli theorem shows the compactness.

Particularly interesting case is when $\gamma=\hat{d}$. This can be concluded from Theorem 2 with $\omega=1$. We state this result as a separate theorem:

Corollary 2. Let $G$ be a $L C A$ group such that $d$ and $\hat{d}$ are dual metrics. Suppose that $\gamma(\xi)=\hat{d}(\xi, \hat{e})$ and $\hat{\mu}$ is upper $\beta$-regular. If $\frac{\beta}{2}<s<\frac{\beta}{2}+1$, then $H_{\gamma}^{s}(G) \hookrightarrow$ $C^{0, \alpha}(G)$, where $\alpha=s-\frac{\beta}{2}$. Moreover, there exists $C>0$ such that the following inequality holds

$$
\|u\|_{C^{0, \alpha}(G)} \leq C\|u\|_{H_{\gamma}^{s}(G)}
$$

for all $u \in H_{\gamma}^{s}(G)$. Furthermore, if $s \geq 1+\beta / 2$ then $H_{\gamma}^{s}(G) \hookrightarrow C^{0, \alpha}(G)$ for all $\alpha \in(0,1)$.

As a corollary, we have the corresponding result in $p$-adic analysis ${ }^{2}$.

Corollary 3. Let $s=\alpha+n / 2$ with $\alpha \in(0,1)$. Then,

$$
H_{d}^{s}\left(\mathbf{Q}_{p}^{n}\right) \hookrightarrow C^{0, \alpha}\left(\mathbf{Q}_{p}^{n}\right)
$$

where $d$ is a standard $p$-adic metric.

The above corollary is in fact an improved version of the embedding proved by Rodríguez-Vega and Zúñiga-Galindo in [24].

3.3. Moser-Trudinger inequality. In this subsection we shall investigate the limiting case of the embedding when $s=\frac{\beta}{2 \omega}$. Those type of results refer to the socalled Moser-Trudinger inequality (see $[21,28]$ for Sobolev spaces in the Euclidean setting, [29] for Sobolev spaces on complete noncompact Riemannian manifolds, [2] for Sobolev spaces in metric measure setting and [30] for Sobolev spaces on the Heisenberg group $\mathbf{H}^{n} 3$ ).

\footnotetext{
${ }^{2}$ The dual group to $\mathbf{Q}_{p}^{n}$ is $\mathbf{Q}_{p}^{n}$.

${ }^{3}$ The Heisenberg group $\mathbf{H}^{n}$ is not abelian.
} 
Lemma 2. Let $G$ be a $L C A$ group such that the measure $\hat{\mu}$ is upper $\beta$-regular and $\gamma \in \Gamma_{A}(\omega)$. Then there exists $C=C\left(\omega, \beta, A, D_{\beta}\right)$ such that for all $u \in H_{\gamma}^{\frac{\beta}{2 \omega}}(G)$ and all $p \geq 2$

$$
\|u\|_{L^{p}(G)} \leq C \sqrt{p}\|u\|_{H_{\gamma}^{\frac{\beta}{2 \omega}}(G)} .
$$

Proof. The proof falls naturally into two steps.

Step 1. For $p=2$ the lemma is obvious. Next, let $p>2$ and set $s_{p}=\frac{\beta}{2 \omega} \frac{p-1}{p}$. Note that

$$
\frac{\beta}{2 \omega} \frac{p-2}{p}<s_{p}<\frac{\beta}{2 \omega}
$$

hence it is sufficient to show that

$$
H_{\gamma}^{\frac{\beta}{2 \omega}}(G) \hookrightarrow H_{\gamma}^{s_{p}}(G) \hookrightarrow L^{p}(G) .
$$

The left embedding trivially holds with constant of embedding equal 1. Next, we show the right one. For this purpose let us put $\alpha_{p}=\frac{p s_{p}}{p-2}>s_{p}$. Note that $\alpha_{p}>\frac{\beta}{2 \omega}$, thus by Lemma 1 we have $\frac{1}{1+\gamma^{2}(.)} \in L^{\alpha_{p}}\left(G^{\wedge}\right)$. Moreover, since

$$
\alpha_{p}^{*}=\frac{2 \alpha_{p}}{\alpha_{p}-s_{p}}=\frac{\frac{2 p s_{p}}{p-2}}{\frac{p s_{p}}{p-2}-s_{p}}=p,
$$

by virtue of Theorem 3.5 in [13] we get that for all $p \geq 2$

$$
H_{\gamma}^{s_{p}}(G) \hookrightarrow L^{p}(G)
$$

and

$$
\|u\|_{L^{p}(G)} \leq\left\|\frac{1}{1+\gamma^{2}(.)}\right\|_{L^{\alpha_{p}}\left(G^{\wedge}\right)}^{\frac{s_{p}}{2}}\|u\|_{H_{\gamma}^{s_{p}}(G)} \leq\left\|\frac{1}{1+\gamma^{2}(.)}\right\|_{L^{\alpha_{p}\left(G^{\wedge}\right)}}^{\frac{s_{p}}{2}}\|u\|_{H_{\gamma}^{\frac{\beta}{2 \omega}}(G)} .
$$

Step 2. We estimate the quantity $\left\|\frac{1}{1+\gamma^{2}(.)}\right\|_{L^{\alpha_{p}}\left(G^{\wedge}\right)}^{\frac{s_{p}}{2}}$. Taking $R=1$ in Lemma 1 we have

$$
\left\|\frac{1}{1+\gamma^{2}(.)}\right\|_{L^{\alpha_{p}\left(G^{\wedge}\right)}}^{\alpha_{p}} \leq C_{A, \alpha} D_{\beta}\left[\max \left(\frac{\hat{\mu}_{G}(\{\hat{e}\})}{D_{\beta}}, 1\right)+\frac{2^{\beta}}{1-2^{\beta-2 \alpha_{p} \omega}}\right] .
$$

Since

$$
\beta-2 \alpha_{p} \omega=\frac{\beta}{2-p}, \quad \alpha_{p}=\frac{\beta}{2 \omega} \frac{p-1}{p-2}, \quad \frac{s_{p}}{2 \alpha_{p}}=\frac{p-2}{2 p},
$$

we obtain

$$
\begin{aligned}
\left\|\frac{1}{1+\gamma^{2}(.)}\right\|_{L^{\alpha_{p}}\left(G^{\wedge}\right)}^{\frac{s_{p}}{2}} & \leq \max \left(1, A^{-s_{p}}\right) D_{\beta}^{\frac{s_{p}}{2 \alpha}}\left[\max \left(\frac{\hat{\mu}_{G}(\{\hat{e}\})}{D_{\beta}}, 1\right)+\frac{2^{\beta}}{1-2^{\beta-2 \alpha_{p} \omega}}\right]^{\frac{s_{p}}{2 \alpha_{p}}} \\
& =\max \left(1, A^{-\frac{\beta}{2 \omega} \frac{p-1}{p}}\right) D_{\beta}^{\frac{p-2}{2 p}}\left[\max \left(\frac{\hat{\mu}_{G}(\{\hat{e}\})}{D_{\beta}}, 1\right)+\frac{2^{\beta}}{1-2^{\frac{\beta}{2-p}}}\right]^{\frac{p-2}{2 p}}
\end{aligned}
$$




$$
\begin{aligned}
& \leq \max \left(1, A^{-\frac{\beta}{2 \omega}}\right) \max \left(1, D_{\beta}\right)\left[\max \left(\frac{\hat{\mu}_{G}(\{\hat{e}\})}{D_{\beta}}, 1\right)^{\frac{p-2}{2 p}}+\frac{2^{\frac{\beta(p-2)}{2 p}}}{\left(1-2^{\frac{\beta}{2-p}}\right)^{\frac{p-2}{2 p}}}\right] \\
& \leq \max \left(1, A^{-\frac{\beta}{2 \omega}}\right) \max \left(1, D_{\beta}\right)\left[\max \left(\frac{\hat{\mu}_{G}(\{\hat{e}\})}{D_{\beta}}, 1\right)+\frac{2^{\frac{\beta}{2}}}{\left(1-2^{\left.\frac{\beta}{2-p}\right)^{\frac{p-2}{2 p}}}\right]}\right.
\end{aligned}
$$

where the elementary inequality $(a+b)^{\delta} \leq a^{\delta}+b^{\delta}$ with $0 \leq \delta \leq 1$ has been applied. In order to finish the proof we need to show that

$$
\left\|\frac{1}{1+\gamma^{2}(.)}\right\|_{L^{\alpha_{p}\left(G^{\wedge}\right)}}^{\frac{s_{p}}{2}} \leq C \sqrt{p},
$$

with $C$ independent of $p$. For this purpose, let us define the following map

$$
g_{\beta}(p)=\frac{2^{\frac{\beta}{2}}}{\left(1-2^{\frac{\beta}{2-p}}\right)^{\frac{p-2}{2 p}} .}
$$

By a straightforward calculation we get

$$
\lim _{p \rightarrow+\infty} \frac{g_{\beta}(p)}{\sqrt{p}}=\frac{2^{\frac{\beta}{2}}}{\sqrt{\beta \log 2}} .
$$

Hence, there exists $C_{1}(\beta)$ such that

$$
\frac{g_{\beta}(p)}{\sqrt{p}} \leq C_{1}(\beta)
$$

for all $p \geq 2$. Thus, we proved inequality (4) and it completes the proof of the lemma.

Now, we are in position to state and proof the main result of this subsection.

Theorem 3. Let $G$ be a $L C A$ group and the measure $\hat{\mu}$ is upper $\beta$-regular. Suppose that $\gamma \in \Gamma_{A}(\omega)$, then there exist constants $C$ and $\alpha>0$ such that

$$
\sup _{u \in H_{\gamma}^{\frac{\beta}{2 \omega}}(G)} \int_{G}\left(e^{\alpha\left(\frac{u(x)}{\|u\|}\right)^{2}}-1\right) d \mu_{G}(x) \leq C,
$$

where $\|u\|=\|u\|_{H_{\gamma}^{\frac{\beta}{2 \omega}}(G)}$.

Proof. Let us take $u \in H_{\gamma}^{\frac{\beta}{2 \omega}}(G)$. If we define $v=\frac{u}{\|u\|}$, then by Lemma 2 we have

$$
\begin{aligned}
\int_{G}\left(e^{\alpha v^{2}(x)}-1\right) d \mu_{G}(x) & =\int_{G} \sum_{k=1}^{\infty} \frac{1}{k !}\left(\alpha|v(x)|^{2}\right)^{k} d \mu_{G}(x)=\sum_{k=1}^{\infty} \frac{\alpha^{k}}{k !}\|v\|_{L^{2 k}(G)}^{2 k} \\
& \leq \sum_{k=1}^{\infty} \frac{\alpha^{k}}{k !}(C \sqrt{2 k})^{2 k}=\sum_{k=1}^{\infty} \frac{\left(2 \alpha C^{2} k\right)^{k}}{k !} .
\end{aligned}
$$

Now, if we take $\alpha>0$ such that $2 \alpha C^{2}<e^{-1}$, then using the Stirling's formula we get that the last series is convergent. This completes the proof.

Let us mention an important consequence of the theorem. 
Theorem 4. Let $G$ be a compact abelian group and the measure $\hat{\mu}$ is upper $\beta$-regular. If $\gamma \in \Gamma_{A}(\omega)$, then there exist constants $C>0, \alpha>0$ such that

$$
\int_{G} e^{\alpha\left(\frac{u(x)}{\|u\|}\right)^{2}} d \mu_{G}(x) \leq C
$$

where as before $\|u\|=\|u\|_{H_{\gamma}^{\frac{\beta}{2 \omega}}(G)}$.

As a corollary we obtain the Moser-Trudinger inequality on $\mathbf{Q}_{p}^{n}$. To the best of our knowledge the following result seems to be new.

Corollary 4. Let $d$ be a standard p-adic metric on $\mathbf{Q}_{p}^{n}$. There exist constants $C>0, \alpha>0$ such that

$$
\sup \int_{\mathbf{Q}_{p}^{n}}\left(e^{\alpha(u(x))^{2}}-1\right) d \mu_{G}(x) \leq C,
$$

where the supremum is taken over $u \in \mathbf{Q}_{p}^{n}$ such that $\|u\|_{H_{d}^{\frac{n}{2}}\left(\mathbf{Q}_{p}^{n}\right)}=1$.

\section{The density of the continuous functions in $\boldsymbol{H}_{\gamma}^{s}$}

In this section we shall state and prove the density theorem for spaces defined on general LCA groups. We refer the reader to the paper of Meyers and Serrin [19] for the fundamental result in the problem of density of regular functions in Sobolev spaces. Let us stress that in this section we do not assume that topological groups are metrizable. We start with the following lemma.

Lemma 3. Let $G$ be a $L C A$ group and $1 \leq p<\infty$. If $f \in L^{p}(G)$ and $\phi \in C_{c}(G)$, then $f * \phi \in C(G) \cap L^{p}(G)$.

Proof. First, we shall show that $f * \phi$ is continuous. For this purpose let us fix $\varepsilon>0$. Since the $\operatorname{map}^{4} z \longmapsto L_{z} f$ is a continuous function from $G$ to $L^{p}(G)$, we can find an open neighborhood $U$ of $e_{G}$ such that for any $z \in U$

$$
\left\|L_{z} f-f\right\|_{L^{p}(G)} \leq \frac{\varepsilon}{\|\phi\|_{L^{p^{\prime}(G)}}^{p^{\prime}}} .
$$

Next, let us take $x, y \in G$ such that $-x+y \in U$. By virtue of the Hölder inequality we get

$$
\begin{aligned}
|f * \phi(x)-f * \phi(y)| & =\left|\int_{G}(f(x-z)-f(y-z)) \phi(z) d \mu_{G}(z)\right| \\
& \leq \int_{G}|f(x-z)-f(y-z)|^{p} d \mu_{G}(z) \cdot\|\phi\|_{L^{p^{\prime}(G)}}^{p^{\prime}},
\end{aligned}
$$

where $\frac{1}{p}+\frac{1}{p^{\prime}}=1$. Changing the variables $u=y-z$ and using the fact that $G$ is abelian we have

$$
\begin{aligned}
\int_{G}|f(x-z)-f(y-z)|^{p} d \mu_{G}(z) & =\int_{G}|f(x-y+u)-f(u)|^{p} d \mu_{G}(u) \\
& =\left\|L_{-x+y} f-f\right\|_{L^{p}(G)} .
\end{aligned}
$$

\footnotetext{
${ }^{4}$ The left translation is defined by $L_{x} f(y)=f\left(x^{-1} y\right)$.
} 
Combining inequalities (5)-(7) we get that $f * \phi$ is continuous. For this reason since

$$
\|f * \phi\|_{L^{\infty}(G)} \leq\|f\|_{L^{p}(G)}\|\phi\|_{L^{p^{\prime}(G)}},
$$

we conclude that $f * \phi \in C(G)$. Furthermore, according to the Young inequality for convolution

$$
\|f * \phi\|_{L^{p}(G)} \leq\|f\|_{L^{p}(G)}\|\phi\|_{L^{1}(G)},
$$

we get that $f * \phi \in L^{p}(G)$.

Our main result of this section is the following claim.

Theorem 5. Let $G$ be a LCA group. Then for any $s>0$

$$
{\overline{H_{\gamma}^{s}(G) \cap C(G)}}^{H_{\gamma}^{s}(G)}=H_{\gamma}^{s}(G) .^{5}
$$

Proof. Let us denote by $\mathcal{I}$ the set of all symmetric unit neighbourhoods of group $G$, partially ordered by inclusion. Let $\left(\phi_{j}\right)_{j \in \mathcal{I}}$ be a Dirac net ${ }^{6}$ on $G$ i.e. $\phi_{j} \in C_{c}(G)$ are non-negative, $\left\|\phi_{j}\right\|_{L^{1}(G)}=1$ and for each unit neighbourhood $U$ there exists $j_{0} \in \mathcal{I}$ such that for each $j \geq j_{0} \operatorname{supp} \phi_{j} \subset U$ (see $[9,12]$ for similar considerations). Take any nonzero function $f \in H_{\gamma}^{s}(G)$ and let us fix $\varepsilon>0$. Since $f$ is in $H_{\gamma}^{s}(G)$, there exists a compact set $K$ such that

$$
\int_{G^{\wedge} \backslash K}\left(1+\gamma^{2}(\xi)\right)^{s}|\hat{f}(\xi)|^{2} d \hat{\mu}_{G}(\xi)<\frac{\varepsilon}{8} .
$$

From Lemma 3 we have $f * \phi_{j} \in L^{2}(G) \cap C(G)$. It remains to show that $f * \phi_{j} \in H_{\gamma}^{s}(G)$ and $\left\|f-f * \phi_{j}\right\|_{H_{\gamma}^{s}(G)}<\varepsilon$ for sufficiently large $j$. By the basic properties of the convolution and the Fourier transform we have

$$
\begin{aligned}
& \int_{G^{\wedge}}\left(1+\gamma^{2}(\xi)\right)^{s}\left|\hat{f}(\xi)-\widehat{f * \phi_{j}}(\xi)\right|^{2} d \hat{\mu}_{G}(\xi) \\
& =\int_{G^{\wedge}}\left(1+\gamma^{2}(\xi)\right)^{s}|\hat{f}(\xi)|^{2}\left|1-\widehat{\phi}_{j}(\xi)\right|^{2} d \hat{\mu}_{G}(\xi) \\
& =\int_{K}\left(1+\gamma^{2}(\xi)\right)^{s}|\hat{f}(\xi)|^{2}\left|1-\widehat{\phi}_{j}(\xi)\right|^{2} d \hat{\mu}_{G}(\xi) \\
& \quad+\int_{G^{\wedge} \backslash K}\left(1+\gamma^{2}(\xi)\right)^{s}|\hat{f}(\xi)|^{2}\left|1-\widehat{\phi}_{j}(\xi)\right|^{2} d \hat{\mu}_{G}(\xi)=I_{1}+I_{2} .
\end{aligned}
$$

Since $K \subset G^{\wedge}$ is compact, by the Arzela-Ascoli theorem we conclude that $K$ is equicontinuous. Thus, there exists an open neighbourhood $U$ of $e_{G}$ such that for all $\xi \in K$ and all $x \in U$

$$
|\xi(x)-1|<\sqrt{\frac{\varepsilon}{2\|f\|_{H_{\gamma}^{s}(G)}^{2}}} .
$$

\footnotetext{
${ }^{5}$ It means that $H_{\gamma}^{s}(G) \cap C(G)$ is dense in $H_{\gamma}^{s}(G)$.

${ }^{6}$ The existence of the Dirac net follows from the Urysohn Lemma, [8].
} 
From the definition of $\phi_{j}$ we can find $j_{0}$ such that for $j \geq j_{0} \operatorname{supp} \phi_{j} \subset U$. Consequently,

$$
\begin{aligned}
\left|1-\widehat{\phi}_{j}(\xi)\right| & =\left|\int_{G} \phi_{j}(x) d \mu_{G}(x)-\int_{G} \overline{\xi(x)} \phi_{j}(x) d \mu_{G}(x)\right| \\
& \leq \int_{\operatorname{supp} \phi_{j}} \phi_{j}(x)|1-\xi(x)| d \mu_{G}(x) \\
& \leq \sqrt{\frac{\varepsilon}{2\|f\|_{H_{\gamma}^{s}(G)}^{2}}} \int_{\operatorname{supp} \phi_{j}} \phi_{j}(x) d \mu_{G}(x)=\sqrt{\frac{\varepsilon}{2\|f\|_{H_{\gamma}^{s}(G)}^{2}}} .
\end{aligned}
$$

This gives $I_{1} \leq \frac{\varepsilon}{2}$. Furthermore,

$$
\left|1-\widehat{\phi}_{j}(\xi)\right|^{2} \leq \int_{\operatorname{supp} \phi_{j}} \phi_{j}(x)|1-\xi(x)|^{2} d \mu_{G}(x) \leq 4 \int_{\operatorname{supp} \phi_{j}} \phi_{j}(x) d \mu_{G}(x)=4,
$$

and hence $I_{2} \leq \frac{\varepsilon}{2}$, which proves the theorem.

Acknowledgements. We wish to thank Wilson Zúñiga-Galindo for comments on $p$ adic analysis. Moreover, we thank Enrique Reyes for reading the preliminary version of this manuscript. Some part of this research has been performed during the staying of P. G. in the Universidad de Santiago de Chile. P. G. thanks the Departamento de Matemática y Ciencia de la Computación for its hospitality. Furthermore, the authors wish to thank an anonymous referee for reviewing the original manuscript.

\section{References}

[1] Adams, R. A.: Sobolev spaces. - Academic Press, New York, 1975.

[2] Adimurthi, and P. Górka: Global Trudinger-Moser inequality on metric spaces. - Forthcoming.

[3] Ambrosio, L., and P. Tilli: Topics on analysis in metric spaces. - Oxford Lecture Ser. Math. Appl. 25, Oxford Univ. Press, Oxford, 2004.

[4] Aubin, T.: Some nonlinear problems in Riemannian geometry. Springer-Verlag, Berlin, 1998.

[5] Bahouri, H., C. Fermanian-Kammerer, and I. Gallagher: Analyse de l'espace des phases et calcul pseudo-differential sur le groupe de Heisenberg. - C. R. Math. Acad. Sci. Paris 347:17-18, 2009, 1021-1024.

[6] Bourbaki, N.: Théories spectrales: Chapitres 1 et 2. - Hermann, 1967.

[7] Capogna, L., D. Danielli, S. D. Pauls, and J. Tyson: An introduction to the Heisenberg group and the sub-Riemannian isoperimetric problem. - Progr. Math. 259, Birkhäuser Verlag, Basel, 2007.

[8] Deitmar, A., and S. Echterhoff: Principles of harmonic analysis. - Springer, 2009.

[9] Górka, P.: Pego theorem on locally compact abelian groups. - J. Algebra Appl. 13:4, 2014, $1350143,1-5$.

[10] Górka, P., and T. Kostrzewa: Pego everywhere. - Forthcoming.

[11] Górka, P., T. Kostrzewa, and E. G. Reyes: The Rellich lemma on compact abelian groups and equations of infinite order. - Int. J. Geom. Meth. Mod. Phys. 10:2, 2013, 1220030, 1-11. 
[12] Górka, P., T. Kostrzewa, and E. G. Reyes: Sobolev spaces on locally compact abelian groups: Compact embeddings and local spaces. - Journal of Function Spaces 2014, 2014, Article ID $404738,1-6$.

[13] Górka, P., and E. G. Reyes: Sobolev spaces on locally compact abelian groups and the bosonic string equation. - J. Aust. Math. Soc. 98, 2015, 39-53.

[14] HajŁasz, P., and P. Koskela: Sobolev met Poincaré. - Mem. Amer. Math. Soc. 145:688, 2000.

[15] Hebey, E.: Sobolev spaces on Riemannian manifolds. - Lecture Notes in Math. 1635, SpringerVerlag, Berlin, 1996.

[16] Heinonen, J.: Lectures on analysis on metric spaces. - Universitext, Springer-Verlag, New York, 2001.

[17] Hewitt, E., and K. A. Ross: Abstract harmonic analysis. Volumes I and II. - Grundlehren Math. Wiss., Springer-Verlag, New York-Berlin, 1963 and 1970.

[18] KIM, Y.: A simple proof of the p-adic version of the Sobolev embedding theorem. - Commun. Korean Math. Soc. 25, 2010, 27-36.

[19] Meyers, N. G., and J. Serrin: $H=W$. - Proc. Nat. Acad. Sci. U.S.A. 51, 1964, 1055-1056.

[20] Montgomery, D., and L. Zippin: Topological transformation groups. - Interscience Publishers, New York-London, 1955.

[21] Moser, J.: A sharp form of an inequality by N. Trudinger. - Indiana Univ. Math. 20, 1971, 1077-1092.

[22] Munkers, J. R.: Topology. - Featured Titles for Topology Series, Prentice Hall, Incorporated, 2000.

[23] Robert, A. M.: A course in p-adic analysis. Grad. Texts in Math. 198. Springer-Verlag, New York, 2000.

[24] Rodríguez-VEgA, J. J., and W. A. ZÚÑIGA-Galindo: Elliptic pseudodifferential equations and Sobolev spaces over $p$-adic fields. - Pacific J. Math. 246:2, 2010, 407-420.

[25] Struble, R. A.: Metrics in locally compact groups. - Compositio Math. 28, 1974, 217-222.

[26] Shanmugalingam, N.: Newtonian spaces: an extension of Sobolev spaces to metric measure spaces. - Rev. Mat. Iberoam. 16:2, 2000, 243-279.

[27] Taylor, M. E.: Partial differential equations. Volume I. - Springer-Verlag, 1996.

[28] Trudinger, N. S.: On imbeddings into Orlicz spaces and some applications. - J. Math. Mech. 17, 1967, 473-483.

[29] Yang, Y.: Trudinger-Moser inequalities on complete noncompact Riemannian manifolds. - J. Funct. Anal. 263:7, 2012, 1894-1938.

[30] YANG, Y.: Trudinger-Moser inequalities on the entire Heisenberg group. - Math. Nachr. 287:89, 2014, 1071-1080. 